\title{
CLINICAL CHARACTERISTICS AND COMORBIDITY OF PEDIATRIC TRICHOTILLOMANIA: THE STUDY OF 38 CASES IN CROATIA
}

\author{
Aleksandra Klobučar ${ }^{1}$, Vera Folnegović-Šmalc ${ }^{2}$, Dubravka Kocijan-Hercigonja ${ }^{3}$, \\ Slavica Sović ${ }^{2,4}$ \& Ljiljana Gulić ${ }^{5}$ \\ ${ }^{I}$ Department of Pediatrics, Department of Child and Adolescent Psychiatry, \\ Children's Hospital Zagreb, Zagreb, Croatia \\ ${ }^{2}$ University of Zagreb, School of Medicine, Zagreb, Croatia \\ ${ }^{3}$ Clinic of Neurology and Psychiatry Kocijan / Hercigonja, Zagreb, Croatia \\ 4 "Andrija Stampar" School of Public Health, Zagreb, Croatia \\ ${ }^{5}$ Psychiatric Hospital for Children and Adolescents, Zagreb, Croatia
}

received: 19.12.2017;

revised: 12.2.2018;

accepted: 19.2.2018

\section{SUMMARY}

Background: The main goal of this study was to analyse and show clinical characteristics and psychiatric comorbidity in 38 participants aged between 10 and 17 with DSM-IV diagnoses of Trichotillomania (TTM) that we were treating at Children's Hospital Zagreb from 2008 to 2017.

Subjects and methods: We analyzed the data obtained from semi-structured interviews by the criteria of DSM-IV, Youth Self Report (YSR) (Achenbach \& Rescorla 2001) and survey that we created.

Results: From 38 participants 21 were girls. The activities during which the participants state that they mostly pull hairs are as follows: doing homework and learning, working on PC, in the toilet, watching TV etc. The most common sites on the body from which participants pulled hair were scalp and among nonscalp sites eyebrows and eyelashes. We found nail biting in more than a half of participants. In 22 participants one or more comorbid disorder has been found, of which ADHD $(n=6)$ and tics $(n=5)$ are most co-occurring disorders. The internalized and externalized problems were nearly evenly represented. Trichophagia was reported by two participants. The results indicate that more than two thirds of participants isolate themselves during hair pulling and half of them try to hide consequences. Median time from the first occurrence of the symptoms to the first visit to a child psychiatrist caused by TTM problem was 9 months (min 5; max 24) what we consider a very long period of time that increased the probability of complications.

Conclusions: Knowledge about this disorder and cooperation among pediatric experts is extremely important for recognizing it at an early stage and starting the treatment especially considering habit-forming mechanism, the burden of an emotional distress and frequent comorbidity. Further research is needed.

Key words: trichotillomania (TTM) - hair pulling disorder (HPD) - children - adolescents - comorbidity

\section{INTRODUCTION}

Trichotillomania (TTM) or hair-pulling disorder is characterized by the repeated pulling of hairs from the body, resulting in significant hair loss. Attempts of reducing or stopping hair pulling are being repeated with considerable clinical suffering and functional deficits (APA 2013). Initially, TTM symptoms usually occur at the beginning and during adolescence. In the fifth edition of the Diagnostic and Statistical Manual of Mental Disorders this disorder was placed in the group together with obsessive-compulsive (OCD) and similar disorders (APA 2013). In DSM-IV it was classified as impulse control disorder (APA 2000). The clinical picture in children and adolescents is often complicated by the presence of comorbidity (Franklin et al. 2008, Harrison \& Franklin 2012, Grant et al. 2017). In this research our main goal was to analyse and show clinical characteristics and psychiatric comorbidity among preadolescents and adolescents with diagnosed TTM.
The adolescent-onset subtype has been related with more psychiatric comorbidity and worse treatment outcome (Odlaug et al. 2012). We also wanted to indicate specific characteristics of our findings that are important in recognizing and treating of this disorder that has been known in psychiatry and dermatology for a long time but conclusions of almost all studies indicate that there hasn't been enough research done especially referring to paediatric population.

\section{SUBJECTS AND METHODS}

We conducted a prospective study assessing clinical characteristics and comorbidity in 38 preadolescent children and adolescents aged between 10 and 17 with DSM-IV diagnoses of TTM, treated at Children's Hospital Zagreb from 2008 to 2017. Criteria for participation in study were: diagnosed TTM by criteria of DSMIV with or without increasing and decreasing tension associated with pulling (criteria B and C), symptoms of 
TTM exists at least six months, intellectual abilities are estimated at least at average, the participant did not suffer from any other acute or chronic disease nor takes any drugs that can significantly influence on any functional domain. After 2013 all subjects met the DSM-V criteria for TTM. Out of total 44 preadolescents and adolescents diagnosed with TTM in our hospital during study period of time, six of them did not meet the criteria to participate in the study because of their intellectual abilities $(n=2)$; did not give consent to participate in the study $(n=2)$; were unavailable $(n=2)$. Finally, we included 38 participants from 10 to 17 years old at the beginning of the treatment.

All participants and their parents were informed about the details of the research and they gave a written consent for participation in the study. The research itself was approved by the Ethical Committee of the hospital and was supervised by the Declaration of Helsinki.

Medical records were reviewed for demographic information and data obtained in standardized clinical procedures and diagnostic measures administered by a clinician. Diagnostic estimations and testings were done by a child psychiatrist respecting the standard procedure and using DSM-IV; Trichotillomania Diagnostic Interview (TDI), a semi-structured interview designed to assess symptoms related to TTM (Rothbaum \& Ninan 1994) and Kiddie-SADS-Present and Lifetime Version (Kaufman et al. 1997), a semi-structured interview designed to assess psychopathology in adolescents using DSM-IV diagnostic criteria. We analysed the data collected by Youth Self Report (YSR), one of the most frequently used questionnaires for evaluation of behavior and emotional problems among preadolescents and adolescents at the age from 11-18 years (Achenbach \& Rescorla 2001). In the questionnaires the raw scores lead to 8 syndrome scales (Anxiety/Depression, Withdrawn/Depressed, Somatic complaints, Social problems, Thought problems, Attention problems, Antisocial behavior, Aggressive behavior) which can be grouped into summary scales (Internalizing problems, Externalizing problems, Total problems). We also analyzed the data obtained from The survey about clinical characteristics that we created to get research material and insight into the clinical presentation of TTM. We divided it into five parts. In the first part the participants stated activities during which they pull out their hairs most often. In the second part they answered yes or no to two questions: "Do you pull out other people's hair? “ and "Do you pull out your pet's hair?". In the third part of the survey they were asked about itching in the area of pulling. In the fourth part they were answering questions about the presence of other people during the hair pulling. In the fifth part they were asked about post-pulling behaviours. For creation of this survey we were following relevant characteristics of hair pulling behavior that are described in the literature.

Data were analysed using software STATISTIKA 13.3 StatSoft, Tulsa, OK, USA. Sociodemographic, clinical characteristics and comorbidity frequencies are presented as median, minimum and maximum for quantitative and absolute frequencies for qualitative variables. The data collected by Youth Self Report (YSR) have been transformed to T-scores in order to compare them with YSR clinical guidelines.

\section{RESULTS}

Sociodemographic data are shown in Table 1 . The median age of onset of the TTM was 11 years (min 10; max 16). The median school success was very good (4) of five point scale from unsatisfactory (1) to excellent (5).

Table 1. Sociodemographic characteristic of preadolescents and adolescents with Trichotillomania $(\mathrm{N}=38)$ according to gender, status of a single child, marital status of parents, birth order, education of mother, education of father and extracurricular activities

\begin{tabular}{|c|c|c|c|}
\hline \multirow{2}{*}{$\begin{array}{l}\text { Sociodemographic } \\
\text { characteristic }\end{array}$} & Values & \multicolumn{2}{|c|}{ Number of participants } \\
\hline & Girls & \multicolumn{2}{|c|}{21} \\
\hline & Boys & \multicolumn{2}{|c|}{17} \\
\hline Single child & No & \multicolumn{2}{|c|}{29} \\
\hline & Yes & \multicolumn{2}{|c|}{9} \\
\hline Birth order & 1 & \multicolumn{2}{|c|}{29} \\
\hline & 2 & \multicolumn{2}{|c|}{6} \\
\hline & 3 & \multicolumn{2}{|c|}{2} \\
\hline & 5 & \multicolumn{2}{|c|}{1} \\
\hline Divorced parents & No & \multicolumn{2}{|c|}{35} \\
\hline & Yes & \multicolumn{2}{|c|}{3} \\
\hline Extra-curricular & No & \multicolumn{2}{|c|}{22} \\
\hline activities & Yes & \multicolumn{2}{|c|}{16} \\
\hline \multirow[t]{2}{*}{ Type of activities } & Sport & \multicolumn{2}{|c|}{9} \\
\hline & Other & \multicolumn{2}{|c|}{7} \\
\hline \multicolumn{2}{|l|}{ Parents education } & Mother & Father \\
\hline \multicolumn{2}{|c|}{ Primary School } & 2 & 2 \\
\hline \multicolumn{2}{|c|}{ Secondary School } & 15 & 15 \\
\hline \multicolumn{2}{|c|}{ High School/College } & 19 & 18 \\
\hline \multicolumn{2}{|c|}{ Master's Degree } & 2 & 3 \\
\hline \multicolumn{2}{|c|}{ Doctorate Degree } & 0 & 0 \\
\hline
\end{tabular}

Most of the patients were directed to a child psychiatrist by paediatric dermatologist $(\mathrm{n}=23)$, paediatricians $(n=8)$, others child psychiatrists $(n=4)$, psychologist $(n=1)$ and two patients came with their parents on their own initiative after they had been informed about the symptoms through the media. Median time from the first occurrence of the symptoms to the first visit to a child psychiatrist caused by TTM problems was 9 months ( $\min 5$; $\max 24$; Mode 9). In eight participants some of co-occurring disorders were diagnosed earlier, and in seven of them certain psychiatric disorders were suspected, so they were all examined by a paediatrician of primary health care, and some of them also by a psychiatrist $(n=7)$ and psychologist $(n=8)$. We checked the diagnoses and we found in two participants with the 
diagnosis of ADHD established at preschool age the increased values for possible manifestation of disorder but we did not find the elements that satisfy the diagnosis criteria. Other diagnoses were confirmed.

\section{Clinical characteristics and comorbidity}

Activities during which hair pulling occurred most frequently are shown in order of frequency (Table 2).

Table 2. Activities during which hair pulling occurred most frequently in preadolescents and adolescents with Trichotillomania (N=38)

\begin{tabular}{lc}
\hline Activities & $\begin{array}{c}\text { Number of } \\
\text { participants }\end{array}$ \\
\hline Doing homework or studying & 25 \\
Playing or using PC, mobile phone or similar & 25 \\
Watching TV & 22 \\
In the toilet & 16 \\
Trying to fall asleep & 12 \\
Driving in the car & 9 \\
Grooming in front of the mirror & 9 \\
Resting & 6 \\
At school during lessons & 6 \\
At school during break & 5 \\
Visiting guests & 5 \\
At the cinema & 5 \\
Talking on the phone & 4 \\
Having a bath & 3 \\
\hline
\end{tabular}

Four participants gave positive answer to the question if they pulled other person's hair. Three of them pulled their sibling's hair and one of them his grandmother's hair. One participant pulled his pet's hair. Seven participants felt itching in the area of hair pulling but it was not connected with hair pulling behaviour. Three of them reported that they felt itching before, and seven of them reported that they felt itching after pulling out hairs. 31 participants reported how they pulled hairs only when they were alone, six reported that they did it in the presence of the closest members of family, and one in the presence of other persons that are not members of family. Two participants reported that they chew pulled hairs, and sometimes swallow them. Twelve participants whirl their hairs between fingers, stretch them and seven rub them around their mouth and lick them. Four participants collect their hairs on hidden places. Twenty participants try to collect their hairs and then they scatter them around or throw them in the toilet or garbage in order to hide the traces.

Most frequently, the participants pull their hair from their scalps $(n=34)$, more detailed results are shown in Table 3.

The measuring results of internalized and externalized problems are shown in Table 4. The results are shown distributed to "Normal" which are in the range of normative values, "Border" with the risk of developing a problem and "Clinical" with the biggest risk of developing psychopathology of the problem in question.

Table 3. Hair pulling areas of the body in preadolescents and adolescents with Trichotillomania $(\mathrm{N}=38)$

\begin{tabular}{lc}
\hline Area & Number of participants \\
\hline Scalp & 34 \\
One scalp site & 11 \\
Eyebrows & 13 \\
Eyelashes & 14 \\
Arms & 1 \\
Legs & 1 \\
\hline
\end{tabular}

Table 4. The results of data processing of the questionnaire for estimation of behavior of youth YSR (Youth Self Report) among preadolescents and adolescents with Trichotillomania $(\mathrm{N}=38)$. Distribution of the participants within the Normative, the Border, and the Clinical range with respect to the YSR/11-18 scales*

\begin{tabular}{lcccc}
\multicolumn{1}{c}{ Syndrome scales } & \multicolumn{4}{c}{ Range with respect to the Youth Self Report scales } \\
& Normal & Border & Clinical & Total number of participants \\
\hline Anxious/depressed & 34 & 3 & 1 & 38 \\
Withdrawn/depressed & 35 & 2 & 1 & 38 \\
Somatic complaints & 35 & 3 & 0 & 38 \\
Social problems & 35 & 1 & 2 & 38 \\
Thought problems & 33 & 4 & 1 & 38 \\
Attention problems & 35 & 3 & 0 & 38 \\
Rule-breaking behavior & 34 & 3 & 1 & 38 \\
Aggressive behavior** & 34 & 0 & 3 & 37 \\
\hline Summary scales & & & & 38 \\
\hline Internalizing problems & 35 & 2 & 1 & 37 \\
Externalizing problems** & 34 & 0 & 3 & 38 \\
Other problems & 36 & 0 & 2 & 37 \\
Total score** & 33 & 2 & 2 & \\
\hline
\end{tabular}

*Analysis included the questioners of three participants at the age from 10-11 years;

** One participant did not fill in the questionnaire correctly in this subscale 
Table 5. Diagnosed psychiatric/neurodevelopmental comorbidity among preadolescents and adolescents with Trichotillomania $(\mathrm{N}=38)$

\begin{tabular}{lc}
\hline Comorbidity; TTM (N=38) & $\begin{array}{c}\text { Number of } \\
\text { participants }\end{array}$ \\
\hline ADHD & 6 \\
Tic & 5 \\
Anxiety disorder & 3 \\
Conduct disorder & 3 \\
Depression & 1 \\
Dysthymia & 2 \\
Obsessive - compulsive disorder & 1 \\
Excoriation disorder & 1 \\
Eating disorder & 1 \\
Specific learning difficulties & 1 \\
Total* & 24 \\
\hline *Two participants were diagnosed with two comorbid \\
disorders, one with ADHD and conduct disorder and one \\
with ADHD and specific learning difficulties
\end{tabular}

In 22 participants comorbidity was diagnosed, with one or two psychiatric and/or neurodevelopmental disorders (Table 5). Considerable number of participants $(n=23)$ bite nails.

\section{DISCUSSION}

The obtained results support researches in which there was no significant difference in representation by gender among children and adolescents with TTM (Reeve et al. 1992, Sathe et al. 2016). Going to a child psychiatrist mostly followed the previous examination by the dermatologist in our hospital, who suspected TTM after examining the patient. Our research also indicates that in 15 out of 38 participants some psychiatric disorders have been suspected or diagnosed even before TTM was diagnosed. All those patients were observed by a primary health care paediatrician, and some of them also by psychiatrist or a psychologist. This research indicates that the difficulties of a recognizing the problem at an early stage exist regardless of the already developed clinical presentation, for which there are more possible reasons. The results of this research indicate that more than two thirds of participants isolate themselves mostly during hair pulling and reluctantly admit hair pulling. A half of participants try to hide the consequences which makes recognizing the problem by parents or experts even harder. It can be that some of participants did not have any additional problems or in the family there are cultural, sociological or some other obstacles together with the resistance to a psychiatric treatment of the problem. It can also be that the experts in paediatric medicine, including mental health professionals, do not often meet this disorder or they are not enough acquainted with this disorder. New technologies enable those who suffer from TTM to seek information and look for help among support groups via Internet, rather than turning to the professionals for help. Of course, it could be useful but we should not underestimated timely experts help. Our results indicates that TTM is not easily recognized, and if so, one may say that there is the problem in systematic approach to this disorder including level of information, understanding and other possible obstacles among the professionals and wider.

In our sample ADHD and tics are the most frequent comorbidity. Links between TTM and ADHD primarily refer to etiopathogenesis and phenomenological aspects of impulsivity and distraction. Some researches indicate the frequency of comorbidity of TTM and ADHD from 9\% to 16.5\% (Christenson 1991, Malhotra et al. 2008, Brennan 2016, Rozenman et al. 2016) and in one research very high frequency of $44.4 \%$ was shown (Sathe et al. 2016). The majority of links between these disorders still remains unanswered. In certain researches comorbidity of TTM and tics are estimated at 6-10\% (Hanna 1997, Rozenman et al. 2016, Brennan et al. 2016). One research indicates that TTM may be more similar to tic disorders than to OCD in terms of cooccurring anxiety, depression and externalizing symptoms (Rozenman et al. 2016). Frequency of comorbidity between TTM and these two disorders as well as the data found in our research, especially those about the number of the participants with ADHD that have been diagnosed before the occurrence of TTM, indicate that it would be reasonable to continue research of possible higher risk for the occurrence of TTM in children with ADHD or of some other correlations in etiopathogenesis of these disorders. More than a half of the participants bite nails that supports such results in the literature (Stein et.al. 2010). Links might be looked for and explained within the domain of autodestruction, compulsive repetition and habit (Ghanizadeh 2011, McGuire et al. 2012). Considering the known data (Lewin et al. 2009, Grant et al. 2017) we expected higher result in prevalence of internalized problems and diagnosed disorders in comorbidity with TTM. The study indicates that there is nearly the same prevalence of internalized and externalized problems. This might correspond to comorbidity that has been diagnosed. Positive connections between internalized and externalized problems have been seen on many samples (Achenbach \& Rescorla 2001). One of the limitations of this brief report is not expanding the analysis. TTM can cause serious emotional distress which can make children and adolescents more vulnerable and it can induce especially the development of internalized as well as externalized problems and behaviour (Franklin et al. 2008, Harrison \& Franklin 2012). Similarly, children with certain psychiatric disorders may have an increased risk of developing TTM or there could be some other correlations in pathogenesis of these different disorders. The data was collected at the beginning of the treatment and further research is needed. 
Hair pulling during sitting activities and when the participants are alone has been often reported in the literature (Tay et al. 2004) and our research supports the data that preadolescents and adolescents pull their hairs mostly during these activities. In 16 participants staying in toilet as an activity during which they pull their hairs has been marked. It might be that they do it there in order to be alone, in privacy so that they can pull their hairs without being disturbed or checked. Activities like combing hair, applying, makeup and other activities concerning the body can be a part of ritual by these participants. Different sensory stimuli have been described as possible triggers for hair pulling in TTM (Bohne et al. 2005, Flessner et al. 2009). In the literature driving a car by adults has been described as an activity during which they pull hairs, but we have not found the quotes or possible explanations that we would connect with our result which indicates that nine out of 38 participants state that one of activities during which they mostly pull their hairs is driving in a car. It is not clear how to explain this result, there is a series of significant risk factors; from sitting activities, boredom or waiting up for sensory stimuli during driving (Rogers \& Luby 2011).

Our research supports most of findings from the literature which indicates that children and adolescents mostly pull hair, then eyebrows and eyelashes but rarely hairs from other parts of the body (axillary, pubic regions) (Franklin et al. 2008, Walther et al. 2014). Four participants pull other person's hair and one pulls his dog`s hairs which makes us think in different directions, one of which is psychodynamic thinking about the release of aggressive impulses, and also about neurobiological and neurocognitive aspects that refer to compulsion and forming a pathological habit. Most of the participants are the first-born children which can be significant for psychodynamic approach in exploration of emotional development.

Although the observation was thorough and well planned certain results are based on self-report data which set some limitations on the research. In comparison with the number of participants in previous researches of TTM, based on the literature on TTM, our sample size is representative enough, but the main limitation of this research is not having sample that is large enough, which disables us in making generalized conclusions in the interpretation of the results and it does not provide adequate predictive value. Consequently we neither researched statistical significance of differences in certain links of clinical characteristics among TTM which occurs with comorbidity and TTM without comorbidity, nor the cause and consequence relationships among certain variables.

\section{CONCLUSION}

Former researchers of clinical characteristic and comorbidity have significantly contributed to better understanding and more efficient treatment of this disorder.
This research indicates that there are some difficulties of recognizing the problem at an early stage regardless of the intensity of already developed clinical picture, for which there are more possible reasons. Cooperation among experts and knowledge about this disorder is extremely important for recognizing it at an early stage and starting the treatment especially considering habitforming mechanism, the burden of an emotional distress and frequent comorbidity. This cooperation should be supported by interdisciplinary education and the information about this disorder should be easily available. Psychiatric/neurodevelopmental comorbidity complicate diagnostic procedure and also make the choice of psychotherapeutic approach and treatment with medications difficult. The researches of these segments, if we exclude those on Internet samples, were mostly conducted on small number of participants, so further research is needed due to the complexity of it and differences among individuals in clinical characteristics and comorbidity.

\section{Acknowledgements: None.}

\section{Conflict of interest: None to declare.}

\section{Contribution of individual authors:}

Aleksandra Klobučar: the idea for the study, wrote the paper, selection of literature, discussion contribution and conclusion;

Vera Folnegović-Šmalc: the idea for the study;

Dubravka Kocijan-Hercigonja: discussion contribution and conclusion, read and revised manuscript content;

Slavica Sović: discussion contribution and conclusion, read and revised manuscript content;

Ljiljana Gulić: selection of literature, read and revised manuscript content.

\section{References}

1. Achenbach TM \& Rescorla LA: Manual for the ASEBA School-Age Forms \& Profiles. Burlington, VT: University of Vermont, Research Center for Children, Youth, \& Families, 2001

2. American Psychiatric Association: Diagnostic and statistical manual of mental disorders. $4^{\text {th }} \mathrm{ed}$. American Psychiatric Association. Washington, DC, 2000

3. American Psychiatric Association: Diagnostic and statistical manual of mental disorders. $5^{\text {th }}$ ed. American Psychiatric Association, Washington, DC, 2013

4. Bohne A et al.: Visuospatial abilities, memory, and executive functioning in trichotillomania and obsessive-compulsive disorder. Journal of Clinical and Experimental Neuropsychology 2005; 27:385-399

5. Brennan E, Francazio S, Gunstad $J$ \& Flessner $C$ : Inhibitory Control in Pediatric Trichotillomania (Hair Pulling Disorder): The Importance of Controlling for Age and Symptoms of Inattention and Hyperactivity. Child Psychiatry Hum Dev 2016; 47:173-182 
6. Christenson GA, Pyle RL \& Mitchell JE: Estimated lifetime prevalence of trichotillomania in college students. J Clin Psychiatry 1991; 52:415-417

7. Diefenbach GJ, Mouton-Odum S, Stanley MA: Affective correlates of trichotillomania. Behav Res Ther 2002; 40:1305-1315

8. Flessner CA, Woods DW, Franklin ME, Keuthen NJ \& Piacentini J: Cross-sectional study of women with trichotillomania: a preliminary examination of pulling styles, severity, phenomenology, and functional impact. Child Psychiatry Hum Dev 2009; 40:153-167

9. Franklin ME et al.: The Child and Adolescent Trichotillomania Impact Project: descriptive psychopathology, comorbidity, functional impairment, and treatment utilization. J Dev Behav Pediatr 2008; 29:493-500

10. Ghanizadeh A: Nail Biting; Etiology, Consequences and Management. Iranian Journal of Medical Sciences 2011; 36:73-79

11. Grant JE, Redden SA, Leppink EW \& Chamberlain SR: Trichotillomania and Co-Occurring Anxiety. Comprehensive psychiatry 2017; 72:1-5

12. Hanna GL: Trichotillomania and related disorders in children and adolescents. Child Psychiatry Hum Dev 1997; 27:255-268

13. Harrison JP \& Franklin ME: Pediatric trichotillomania. Curr Psychiatry Rep 2012; 14:188-196

14. Kaufman J, Birmaher B, Brent D, Rao U, Flynn C, Moreci $P$ et al.: Schedule for affective disorders and schizophrenia for school-age children-present and lifetime version (K-SADS-PL): initial reliability and validity data. J Am Acad Child Adolesc Psychiatry 1997; 36:980-8

15. Lewin $A B$ et al.: Depression, anxiety, and functional impairment in children with trichotillomania. Depress Anxiety 2009; 26:521-7

16. Malhotra S, Grover S, Baweja $R$ \& Bhateja G: Trichotillomania in children. Indian Pediatr 2008; 45:403-5
17. McGuire JF, Kugler BB, Park JM, Horng B, Lewin AB, Murphy TK et al.: Evidence-based assessment of compulsive skin picking, chronic tic disorders and trichotillomania in children. Child Psychiatry Hum Dev 2012; 43:855-88

18. Odlaug BL, Chamberlain SR, Harvanko AM \& Grant JE: Age at onset in trichotillomania: clinical variables and neurocognitive performance. Prim Care Companion CNS Disorder 2012; 14:1-13

19. Reeve EA, Bernsten DA \& Christenson GA: Clinical characteristics and psychiatric comorbidity in children with trichotillomania. J Am Acad Child Adolesc Psychiatry 1992; 31:132-138

20. Rogers $C \&$ Luby J: Sensory over-responsivity: a diagnosis whose time has come? J Am Acad Child Adoles Psychiatry 2011; 50:1205-1207

21. Rothbaum BO \& Ninan PT: The assessment of trichotillomania. Behav Res Ther 1994; 32:651-662

22. Rozenman M, Peris TS, Gonzalez A \& Piacentini J: Clinical characteristics of pediatric trichotillomania: Comparisons with obsessive-compulsive and tic disorders. Child Psychiatry Hum Dev 2016; 47:124-132

23. Sathe $H$ et al.: Childhood onset trichotillomania: a retrospective analysis of 78 cases visiting tertiary general hospital. Int J Contemp Pediatr 2016; 3:1284-1287

24. Stein DJ et al.: Trichotillomania (hair pulling disorder), skin picking disorder, and stereotypic movement disorder: toward DSM-V. Depress Anxiety 2010; 27:611-626

25. Tay YK, Levy ML \& Metry DW: Trichotillomania in childhood: case series and review. Pediatrics 2004; 113:494-498

26. Walther MR, Snorrason I, Flessner CA, Franklin ME, Burkel $R \&$ Woods DW: The trichotillomania impact project in young children (TIP-YC): clinical characteristics, comorbidity, functional impairment and treatment utilization. Child Psychiatry Hum Dev 2014; 45:24-31

27. Woods DW \& Houghton DC: Diagnosis, Evaluation, and Management of Trichotillomania. The Psychiatric clinics of North America 2014; 37:301-317
Correspondence:

Aleksandra Klobučar, MD, Child and Adolescent Psychiatrist

University Department of Paediatrics, Department of Child

and Adolescent Psychiatry, Children's Hospital Zagreb

Klaićeva 16, 10000 Zagreb, Croatia

E-mail: sandraklobucar@gmail.com 